\title{
Randomized Comparative Study Between Laparoscopic Transabdominal Pre-Peritoneal Versus Totally Extraperitoneal Approach in Inguinal Hernia Repair
}

\author{
Abdelaziz Osman Elhendawy, Osama Hasan Abd-Raboh, Taha Ahmad Ismail, \\ Abdelmonem Ahmad Nagy
}

Gastrointestinal and Laparoscopic Surgery Department, Faculty of Medicine, Tanta University, Tanta, Egypt

Email address:

dr_osama_hassan@med.tanta.edu.eg(O.H. Abd-Raboh)

\section{To cite this article:}

Abdelaziz Osman Elhendawy, Osama Hasan Abd-Raboh, Taha Ahmad Ismail, Abdelmonem Ahmad Nagy. Randomized Comparative Study Between Laparoscopic Transabdominal Pre-Peritoneal Versus Totally Extraperitoneal Approach in Inguinal Hernia Repair. Advances in Surgical Sciences. Vol. 6, No. 1, 2018, pp. 1-6. doi: 10.11648/j.ass.20180601.11

Received: January 13, 2018; Accepted: January 26, 2018; Published: February 19, 2018

\begin{abstract}
Background: Whether totally extraperitoneal inguinal hernia repair (TEP) is associated with better outcomes than transabdominal preperitoneal inguinal hernia repair (TAPP) continues to be a matter of debate. The objective of this study is to compare outcomes between patients undergoing TEP or TAPP. Methods: This prospective randomized comparative study was carried out in Gastrointestinal and Laparoscopic Surgery Unit, General Surgery Department, Tanta University Hospitals from May 2016 to May 2017, on 30 patients with inguinal hernia, divided into 2 equal groups: Group I: subjected to Transabdominal Pre-peritoneal (TAPP) inguinal hernia repair (15 cases). Group II: subjected to Totally Extraperitoneal (TEP) inguinal hernia repair (15 cases). Results: The mean age was 47.8 \pm 10.4 ranged from 19-65 years. All patients were presented with indirect inguinal hernia. All cases were male except one female patient. Operative time was $151.7 \pm 24.8$ in TAPP approach in comparison to $88.42 \pm 30.6$ minutes in TEP approach. Post-operative scrotal edema was higher in TAPP approach (9 cases) in comparison to TEP approach (3cases). Postoperative Hematoma and ileus were higher in TAPP technique (2cases). Postoperative Surgical emphysema was higher in TEP technique (3cases). Hospital stay in TAPP approach was longer than TEP approach. There was no difference regarding wound infection, mesh infection and recurrence. Follow up for 6 months of all cases for complication. Conclusion: TEP approach as the laparoscopic procedure of choice for inguinal hernia repair due to short operative time and less hospital stay than TAPP approach.
\end{abstract}

Keywords: Inguinal Hernia, TEP, Totally Extraperitoneal, TAPP, Transabdominal Preperitoneal, Mesh, Fixation, Tacker

\section{Introduction}

Groin hernia repair is one of the most common elective general surgical operations. The lifetime 'risk' of inguinal hernia repair is high, it estimates $27 \%$ for men and $3 \%$ for women [1]. Over the last two centuries there have been a number of techniques described for the repair of inguinal hernias, beginning with the Marcy repair and the milestone Bassini repair up to the present era of laparoscopic inguinal hernia repair. However, minimal access approaches to inguinal hernia repair have added to the ongoing debate over the "best groin hernia repair [2].

Transabdominal preperitoneal (TAPP) approach performed by Arregui requires access to the peritoneal cavity with the placement of mesh through a peritoneal incision. This mesh is placed in the preperitoneal space covering all potential hernia sites in the inguinal region [3, 4]. It has a larger working space than totally extraperitoneal (TEP) approach, with ready access to both groins, and can be done in patients with prior lower abdominal surgery. TAPP is relatively easy to learn [2]. However, TAPP can result in injuries to adjacent intraabdominal organs, adhesions resulting in intestinal obstruction and/or bowel herniation [5]. Recurrence after TAPP appears to be equivalent to open repair [6].

Laparoscopic Totally Extraperitoneal (TEP) approach by McKernan and Laws whom accessed to the preperitoneal 
space without peritoneal violation [7]. TEP is a technically difficult procedure to learn and requires the surgeon to be familiar with laparoscopic anatomy but it has the advantage of direct access to all hernia defects with non-violation of peritoneal cavity [2]. The choice between TEP and TAPP is largely based on the surgeon's preference [8].

\section{Patients and Methods}

This study was a prospective randomized comparative study that was carried out in Gastrointestinal and Laparoscopic Surgery Unit, General Surgery Department, Tanta University Hospital from May 2016 to May 2017, on 30 patients with inguinal hernia, divided into 2 equal groups: Group I: subjected to Transabdominal Pre-peritoneal (TAPP) inguinal hernia repair (15 cases) and Group II: subjected to Totally Extraperitoneal (TEP) inguinal hernia repair (15 cases). The main inclusion criteria were Patients above 18 years' old and Primary inguinal hernias. The exclusion criteria were complicated inguinal hernias, previous preperitoneal operation and patients had severe comorbidities e.g. severe cardiac, hepatic or renal disease.

Surgical Technique:

TAPP technique: It was performed using three ports (one $10 \mathrm{~mm}$ and two $5 \mathrm{~mm}$ ports). The peritoneum was incised 2 $\mathrm{cm}$ above the level of the anterior superior iliac spine and extended medially 3-4 $\mathrm{cm}$ above hernia defect. Dissection of lower peritoneal flap. Sac was dissected and reduced. A large $12 \times 15 \mathrm{~cm}$ piece of polypropylene mesh was placed into the abdomen via the umbilical port and placed preperitoneally. Covering the indirect, direct, and femoral spaces and then stapled to Cooper's ligament and to the superomedial and superolateral corners with 4-6 tackers. The peritoneum was closed with a running 2-0 absorbable suture, the aponeurosis of the umbilical incision was closed with vicryl 0 and the skin was closed with polypropyline $3 / 0$ stitches.

TEP technique: It was performed using three trocars (one $10 \mathrm{~mm}$ and two midline $5 \mathrm{~mm}$ ports). After the ipsilateral anterior rectus sheath was opened, the telescope dissection was made in the preperitoneal space behind the rectus muscle. Dissection of the preperitoneal space then was performed medially across the midline and laterally to the anterior superior iliac spine. The hernia sac was reduced, and a $12 \times 15 \mathrm{~cm}$ polypropylene mesh was placed in this preperitoneal space, covering the inguinal floor and all potential defects. The anterior rectus sheath then was closed with vicryl 0 followed by skin closure.

\section{Results}

The total number of the patients in the present study was 30 patients. 15 patients operated for TAPP approach (group I) giving total 18 operations ( 3 bilateral). 15 patients operated for TEP approach (group II) giving total operations of 19 operations (4 bilateral). The demographic data for both groups are shown in Table 1.
Table 1. Demographic data for both groups.

\begin{tabular}{lllll}
\hline Characteristics & Group I (n=15) & Group II (n=15) & Sig. test & P \\
\hline $\begin{array}{lllll}\text { Age (in years): } \\
\text { Mean } \pm \text { S. D }\end{array}$ & $47.8 \pm 10.4$ & $39.5 \pm 16.4$ & T 1.648 & 0.110 \\
$\begin{array}{llll}\text { Gender: } \\
\text { Male }\end{array}$ & $15(100.0 \%)$ & $14(93.3 \%)$ & $\chi^{2} 1.043$ & 0.309 \\
Female & $0(0.0 \%)$ & $1(6.7 \%)$ & & \\
\hline t: independent sample t test & & & \\
$\chi^{2}:$ Chi square test & & & &
\end{tabular}

As regards the side of the hernia, the results are shown in table 2 .

Table 2. Hernia sides in the studied patient groups.

\begin{tabular}{lllll}
\hline Hernia Side & Group I (n=15) & Group II (n=15) & $\chi^{2}$ & P \\
\hline Right & $6(40.0 \%)$ & $2(13.3 \%)$ & & \\
Left & $6(40.0 \%)$ & $9(60.0 \%)$ & 2.741 & 0.254 \\
Bilateral & $3(20.0 \%)$ & $4(26.7 \%)$ & & \\
\hline
\end{tabular}

\subsection{Operative Time}

A statistically significant difference was present in operative time between both groups. Group I had a longer time than group II. Group I had operative time mean of $151.7 \pm 24.8$ minutes compared to $88.4 \pm 30.6$ minutes in group II as shown in figure 1.

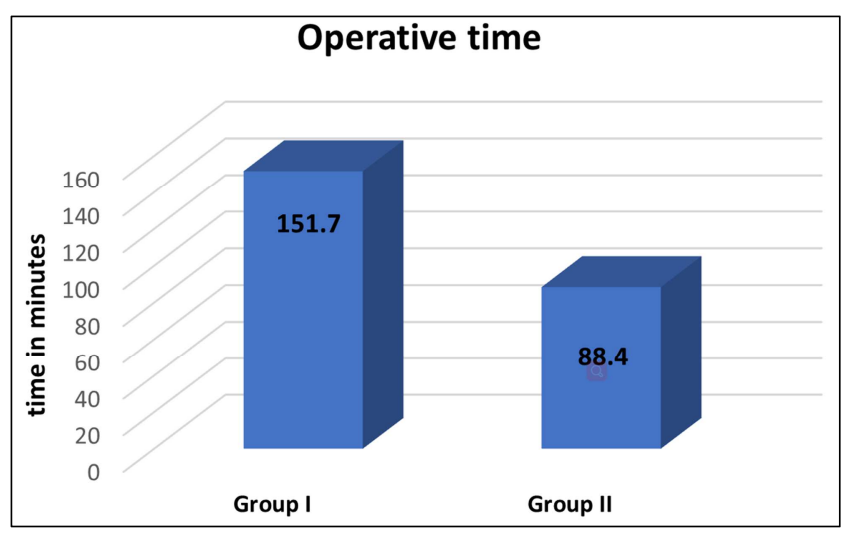

Figure 1. The mean of operative time in minutes among both study groups.

\subsection{Drain insertion}

Closed suction drain was used in 2 cases $(11.1 \%)$ of TAPP group in comparison to 3 cases $(15.8 \%)$ in TEP group with $p$ value $=0.667$ which was statistically insignificant.

\subsection{Intra-Operative Complications}

During this study, only one cases $(6.6 \%)$ was converted from TEP to TAPP procedure due to peritoneal tear during insertion of 2nd trocar. During TEP technique, accidental tear of peritoneum occurred in 4 cases $(26.6 \%)$ which were managed by insertion of Veress needle in the peritoneal cavity to work as a vent. There was no conversion from laparoscopic to open hernia repair in both groups. There was no significant intraoperative bleeding in both groups. 


\subsection{Postoperative Complications}

\section{Postoperative Pain:}

Visual Analogue Pain Scale was used for pain assessment in 1st day after surgery. All patients needed 2 injections of analgesia in the first postoperative day to relieve the pain, and two to three oral doses per day of analgesics till they are pain free. There was no significant difference in postoperative pain in both groups as shown in figure 2.

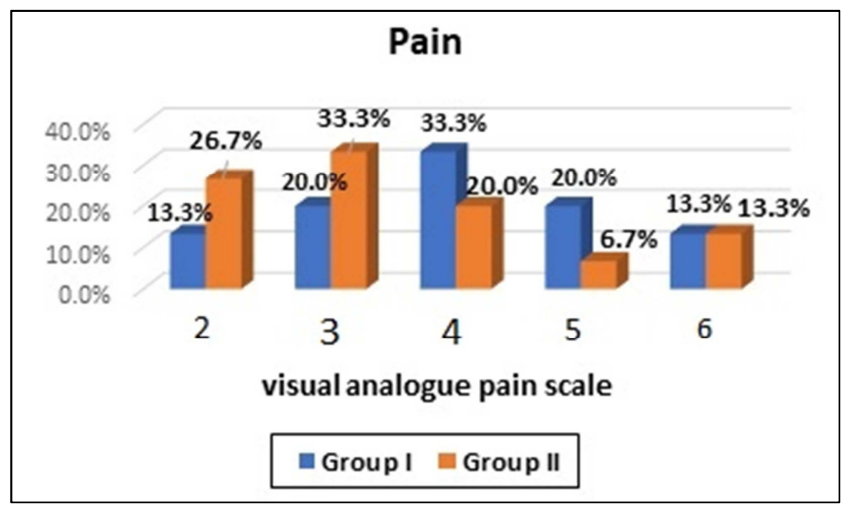

Figure 2. Post-operative pain using visual analogue pain scale at 24 hours postoperative among the study groups.

In this study, 6 cases $(33.3 \%)$ complained from postoperative scrotal edema in TAPP group in comparison to 3 cases $(15.8 \%)$ in TEP group with $\mathrm{p}$ value $=0.214$. This difference was statistically insignificant. 3 cases $(15.8 \%)$ in TEP group presented with postoperative surgical emphysema in comparison with no cases had surgical emphysema in TAPP group with $\mathrm{p}$ value $=0.079$, however it was statistically insignificant. All of these cases were treated conservatively as outpatients. All cases of surgical emphysema resolved within 2 to 3 days postoperative. 2 cases $(11.1 \%)$ of postoperative scrotal hematoma occurred in TAPP group, in comparison to no cases in TEP group with $p$ value $=0.230$ which is statistically insignificant (table 3).

Table 3. Post-operative complications among the study groups.

\begin{tabular}{lllll}
\hline Complication & Group I (n=18) & Group II (n=19) & $\chi^{2}$ & P \\
\hline Scrotal edema & $6(33.3 \%)$ & $3(15.8 \%)$ & 2.727 & 0.214 \\
Surgical & $0(0.0 \%)$ & $3(15.8 \%)$ & 3.093 & 0.079 \\
emphysema & $2(11.1 \%)$ & $0(0.0 \%)$ & 2.232 & 0.230 \\
Hematoma & $2(11.1 \%)$ & $0(0.0 \%)$ & 2.232 & 0.230 \\
Ileus & $0(0.0 \%)$ & $0(0.0 \%)$ & - & - \\
Wound infection & $0(0.0 \%)$ & $0(0.0 \%)$ & - & - \\
Mesh infection & $0(0.0 \%)$ & $0(0.0 \%)$ & - & - \\
Recurrence & & & & \\
\hline
\end{tabular}

$\chi^{2}$ : chi square test

2 cases $(11.1 \%)$ in TAPP group suffered from postoperative ileus which managed by conservative treatment within 2 days, in comparison to no cases in TEP group with $p$ value $=0.230$ indicating statistically insignificant indifference. There were no cases of postoperative wound infection or mesh infection in both groups.

\subsection{Post-Operative Hospital Stay}

In TEP group, hospital stay was shorter than TAPP group as 5 cases $(33.3 \%)$ of TEP group was discharged in 1st day postoperative in comparison to no cases was discharged in 1 st day in TAPP group with $\mathrm{p}$ value $=0.037$ which is statistically significant. In 2 nd day postoperative 8 cases (53.4\%) were discharged in TEP group in comparison to 10 cases $(66.7 \%)$ in TAPP group. In 3 rd day postoperative 2 cases $(13.3 \%)$ of TEP group were discharged in comparison to 5 cases $(33.3 \%)$ in TAPP group as shown in figure 3 .

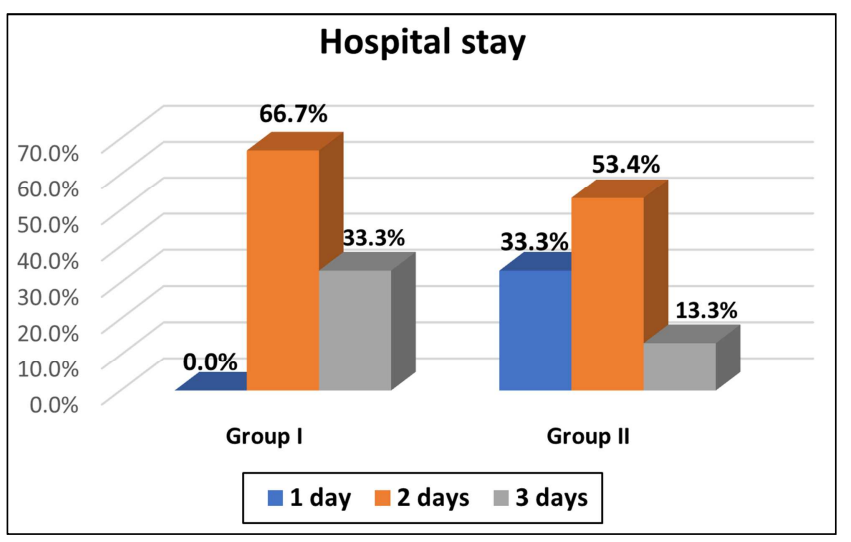

Figure 3. The hospital stay in days among both groups.

\subsection{Follow up}

All of the 30 patients in this study were followed up weekly in the first month. After that, monthly follow up was done for all patients in both groups up to 6 months postoperative. The aim of postoperative follow up was to detect any postoperative complications. No hernia recurrence or chronic groin pain had been detected in any case in both groups during the period of follow up.

\section{Discussion}

Repair of inguinal hernias is one of the most common operations in general surgery, with rates ranging from 10 per 100000 of the population in the United Kingdom to 28 per 100000 in the United States [9]. Surgery is the treatment of choice varying from a nylon darn, Shouldice layered, Lichtenstein mesh to laparoscopic repair [9].

This study evaluated Transabdominal preperitoneal (TAPP) approach and Totally extraperitoneal (TEP) approach with direct dissection and mesh hernioplasty in the treatment of inguinal hernia. Regarding operative time, in this study, the mean operative time in TAPP approach was longer than TEP approach. TAPP approach had a mean operative time of $151.7 \pm 24.8$ minutes compared to $88.4 \pm 30.6$ minutes in TEP approach that was statistically significant ( $\mathrm{p}$ value $=0.001$ ) These results were consistent with Tariq Nawaz who found that: Mean operative time in TEP repair was $45.1 \pm 3.54 \mathrm{~min}$, whereas in TAPP repair was $70 \pm 6.01 \mathrm{~min}(\mathrm{p}=0.000)$ in retrospective study that was conducted on 120 patients for comparison between both techniques [10]. Also Bansal V. K. 
et al., in their study on 314 patients and Köckerling F. et al., on their study on 17,587 patients also found that TAPP technique has a longer time than TEP $[11,12]$.

In contrast, Praveen Kumar et al., found that mean operative time was 97.11 minutes in TAPP and 116.6 minutes in TEP group. The time taken in TEP was significantly more than TAPP group in retrospective study was conducted on 40 patients for comparison between both techniques [13].

During this study, one case $(6.6 \%)$ was converted from TEP to the TAPP procedure due to peritoneal tear during insertion of 2nd trocar, while there was no conversion to open hernia repair. Khan et al., found in their study on 151 patients using TEP technique that $13.9 \%$ of cases were converted to TAPP approach, $7.2 \%$ of cases were converted due to peritoneal tear during TEP procedure and $6.7 \%$ of cases were converted due to hemorrhage and instrumental failure [14]. Umberto Bracale et al., found that there was 6 cases $0.49 \%$ converted to TAPP procedure of total 1209 patients [15]. In this study during TEP technique, accidental tear of peritoneum occurred in 4 cases $(26.6 \%)$ which were managed by insertion of Veress needle in the peritoneal cavity to work as a vent. Lau $\mathrm{H}$. et al., found on their study on 100 patients that the incidence of peritoneal tear was $47 \%$ which was managed by closure of peritoneum by endoscopic stapling or pretied suture loop ligation with no morbidity associated [16]. Chinmay Gandhi et al., found on their study on 30 patients that peritoneal tears occurred in 8 patients $(24.3 \%)$ [17].

None of study patients suffered from significant intraoperative bleeding in both groups of this study. Köckerling F. et al., Found in their study on 17,587 patients that the incidence of bleeding was $53(0.79 \%)$ cases in TEP technique and $108(0.99 \%)$ cases in TAPP technique with $\mathrm{p}$ value $=0.1922$ which was statistically insignificant [12].

In this study, a closed suction drain was put in the peritoneal cavity only in selected cases when there was a large dissected sac or some bleeding, with fear from seroma or hematoma according to surgeon's preference while in TAPP approach, 2 cases $(11.1 \%)$ had a postoperative drain compared to 3 patients $(15.8 \%)$ in TEP approach, that difference was not statistically significant ( $p$-value $=0.173$ ). Tamme recommends routine use of drain in TEP technique, because release of carbon dioxide pressure is followed by bleeding from tiny capillaries, resulting in unpredictable amount of blood collecting in the preperitoneal space.

In this study, the intensity of pain was recorded 24 hours postoperatively. TAPP group had higher pain scores than TEP approach, however that was not statistically significant $\mathrm{p}$ value $=(0.615)$. After follow up for 6 months, there was no cases complained from chronic groin pain in both groups. These results were consistent with the results of the reviews by Umberto Bracale et al., Hamza et al., Dedemadi et al., and Gunal et al., [15, 18-20]. Milind P et al., in their study on 60 patients found that TEP patients were far more comfortable and pain was less than those operated by TAPP. The difference was found to be significant ( $p$ value $<0.0001$ ) [21].

Scrotal edema occurred in 6 patients $(33.3 \%)$ in TAPP approach compared to 3 patients $(15.8 \%)$ in TEP technique and that difference was statistically insignificant $p$-value= 0.214 . These patients were managed conservatively with athletic scrotal support and oral anti-inflammatory tablets. Bansal $\mathrm{P}$ et al., found a significantly higher incidence of postoperative scrotal edema in the TAPP repair group [22]. Asuri Krishna on his study on 100 patients found that the incidence of scrotal edema was significantly higher in the TAPP group (34\%) than in the TEP group $9.4 \%$ with $\mathrm{p}$ value $<0.001$ [2].

In this study, 3 cases $(15.8 \%)$ suffered from postoperative surgical emphysema in TEP group in comparison to no cases in TAPP group with $\mathrm{P}$ value $=0.079$ which was statistically insignificant. All these cases were treated conservatively and surgical emphysema resolved within 2-3 days. Nimesh Verma et al., in his study on 60 patients found 2 cases suffered from Surgical emphysema in TEP group in comparison to 1 case in TAPP group, and these results were statistically insignificant [23].

In this study, 2 cases $(11.1 \%)$ suffered from postoperative hematoma in TAPP group in comparison to no cases in TEP group which was statistically insignificant with $p$ value $=0.230$. Ke Gong et al., found in his study on 164 patients that incidence of postoperative hematoma is higher in TEP group (3.8\%) 3cases in comparison to no cases in TAPP group and this difference wasn't statistically significant [24]. H. Pokorny et al., found in his study that that incidence of postoperative hematoma is higher in TAPP group 7 cases in comparison to 2 cases in TEP group ( $p=$ 0.15 ) and this difference wasn't statistically significant [25]. Milind et al., found that, there was no significant difference between the two techniques in relation to postoperative seroma formation [21].

In this study, 2 cases $(11.1 \%)$ suffered from postoperative ileus in TAPP group in comparison to no cases in TEP group which was statistically insignificant $p$ value $=0.230$. Zirui he et al., found no statistically difference between both groups regarding ileus in their study on 3,203 patients [26].

In this study, the hospital stay in both groups was up to 3 days. As the hospital stay duration in days among TAPP approach group was as the following; 10 patients spent 2 days, and 5 patients spent 3 days. In TEP approach group, 5 patients spent 1 day, 8 patients spent 2 days and 2 patients spent 3 days which was statistically significant (pvalue $=0.037$ ). Asuri Krishna et al., found that there was no statistically significant difference in hospital stay between the two groups $(p=0.056)$. In TEP approach group the postoperative stay was $24.4 \pm 3.2 \mathrm{~h}$ and in TAPP approach group it was $25.2 \pm 5.1 \mathrm{~h}$ with $\mathrm{p}$-value $=0.056$ [2]. Ke Gong et al., found in their study on 164 patients that no significant difference between the TAPP $(\mathrm{p}=0.614)$ and TEP $(\mathrm{p}=$ 0.978) groups [24]. However, Umberto Bracale found that TEP is associated with a significantly shorter hospital stay than TAPP: 0.31 days $(0.082-0.53 ; \mathrm{P}<0.01)$ [15].

No recurrence had occurred in any case of both groups. Leigh Neumayer et al., found that recurrences were more common in the laparoscopic group (in which there were 87 
recurrences among 862 patients $10.1 \%$ than in the open group (in which there were 41 recurrences among 834 patients $4.9 \%$ [27]. However, the reported incidence of recurrence after TEP has been around 1-2\% and after TAPP around $0-3 \%[2,28]$. Ke Gong et al., found in their study on 164 patients that there was no recurrence in both groups [24]. Gunal $\mathrm{O}$ et al., found in their study on 160 patients that one recurrent case in TAPP technique and no recurrent case in TEP technique, there was no statistically significant difference in both groups [20].

In this study, there was no incidence of wound infection in both techniques. Bittner et al., reported 0.1\% mesh infections and $0 \%$ wound infections in 8,050 TAPP procedures in a total of 6,479 patients. Jaime Haidenberg et al., found in their study on 264 patients that one case only suffered from wound infection using TEP technique [29]. In this study, there was no incidence of mesh infection in both techniques. Asuri Krishna et al., found that no incidence of mesh infection in both tecniques in his study on 100 patients [2]. Beverly L Wake et al., found that were very rare and there was no obvious difference between both techniques [30]. In TAPP technique, Schmedt et al., reported $0.07 \%$ infections in 4188 unilateral TAPP procedures and $0 \%$ in 1,336 bilateral procedures. Kapiris et al., reported $0.11 \%$ mesh infections in 3,017 patients, and Leibl et al., reported 3 cases $(0.001 \%)$ in 2,700 patients. Bittner et al., reported $0.1 \%$ mesh infections in 8,050 TAPP procedures in a total of 6,479 patients [31-34]. In TEP technique, Misra M. C. et al., found no incidence of mesh infection in his technique in their study on 56 patients [35].

\section{Conclusion}

In this study, we found that, operative time in TAPP technique was more than TEP technique. Hospital stay in TAPP technique was longer than TEP technique. Both difference was statistically significant. We also found that, postoperative pain, scrotal edema, hematoma and ileus were higher in TAPP technique while surgical emphysema was higher in TEP technique, these differences were statistically insignificant. We recommend TEP approach as the laparoscopic procedure of choice for inguinal hernia repair.

\section{References}

[1] Primatesta P., Goldacre M. J. Inguinal hernia repair: incidence of elective and emergency surgery, readmission and mortality. Int J Epidemiol. 1996; 25 (4):835-839.

[2] Krishna A., Misra M. C., Bansal V. K., Kumar S., Rajeshwari S., Chabra A. Laparoscopic inguinal hernia repair: transabdominal preperitoneal (TAPP) versus totally extraperitoneal (TEP) approach: a prospective randomized controlled trial. Surgical endoscopy. 2012; 26 (3):639-649.

[3] McCormack K, Wake BL, Fraser C, Vale L, Perez J, Grant A. Transabdominal pre-peritoneal (TAPP) versus totally extraperitoneal (TEP) laparoscopic techniques for inguinal hernia repair: a systematic review. Hernia: the journal of hernias and abdominal wall surgery. 2005; 9 (2):109-114.

[4] Bittner R. [Evidence-based TAPP technique]. Chirurg. 2017; 88 (4):281-287.

[5] Wake B. L., McCormack K., Fraser C., Vale L., Perez J., Grant A. M. Transabdominal pre-peritoneal (TAPP) vs totally extraperitoneal (TEP) laparoscopic techniques for inguinal hernia repair. The Cochrane database of systematic reviews. 2005 (1):Cd004703.

[6] O'Reilly E. A., Burke J. P., O'Connell P. R. A meta-analysis of surgical morbidity and recurrence after laparoscopic and open repair of primary unilateral inguinal hernia. Annals of surgery. 2012; 255 (5):846-853.

[7] Schein Moshe. Schwartz's Principles of Surgery, F. Charles Brunicardi, Dana K. Andersen, Timothy R. Billiar, David L. Dunn, John G. Hunter, Jeffrey B. Matthews, Raphael E. Pollock World Journal of Surgery. 2010; 34 (4):871-873.

[8] Sharma A., Chelawat P. Endo-laparoscopic inguinal hernia repair: What is its role? Asian journal of endoscopic surgery. 2017; 10 (2):111-118.

[9] Jenkins J. T., O'Dwyer P. J. Inguinal hernias. Bmj. 2008; 336 (7638):269-272.

[10] 2017 Scientific Session of the Society of American Gastrointestinal and Endoscopic Surgeons (SAGES) Houston, Texas, USA, 22-25 March 2017: Poster Presentations. Surgical endoscopy. 2017; 31 (Suppl 1):137-334.

[11] Bansal V. K., Misra M. C., Babu D., Victor J., Kumar S., Sagar R., Rajeshwari S., Krishna A., Rewari V. A prospective, randomized comparison of long-term outcomes: chronic groin pain and quality of life following totally extraperitoneal (TEP) and transabdominal preperitoneal (TAPP) laparoscopic inguinal hernia repair. Surgical endoscopy. 2013;27.

[12] Köckerling F., Bittner R., Jacob D. A., Seidelmann L., Keller T., Adolf D., Kraft B., Kuthe A. TEP versus TAPP: comparison of the perioperative outcome in 17,587 patients with a primary unilateral inguinal hernia. Surgical endoscopy. 2015; 29 (12):3750-3760.

[13] Kumar Praveen, Kumar Nilkamal. Trans abdominal preperitoneal versus totally extra-peritoneal laparoscopic techniques for inguinal hernia repair: a comparative study. 2016. 2016; 4 (1):4.

[14] Khan Muhammad Siddique, Zeb Junaid, Zaman Amer. Conversion of laparoscopic total extraperitoneal inguinal hernia (TEP) repair to transabdominal preperitoneal (TAPP) repair. Rawal Medical Journal. 2017; 42 (2).

[15] Bracale U., Melillo P., Pignata G., Di Salvo E., Rovani M., Merola G., Pecchia L. Which is the best laparoscopic approach for inguinal hernia repair: TEP or TAPP? A systematic review of the literature with a network metaanalysis. Surgical endoscopy. 2012; 26 (12):3355-3366.

[16] Lau H., Patil N. G., Yuen W. K., Lee F. Management of peritoneal tear during endoscopic extraperitoneal inguinal hernioplasty. Surgical endoscopy. 2002; 16 (10):1474-1477.

[17] GANDHI CHINMAY, DHONDE ASHOK, MOTE DAJIRAM. Pilot study of selective fixation of mesh in laparoscopic extra-peritoneal inguinal hernia repair (TEP). International J of Healthcare and Biomedical Research. 2017; 5 (04):77-84. 
[18] Hamza Y., Gabr E., Hammadi H., Khalil R. Four-arm randomized trial comparing laparoscopic and open hernia repairs. Int J Surg. 2010;8.

[19] Dedemadi G., Sgourakis G., Karaliotas C., Christofides T., Kouraklis G., Karaliotas C. Comparison of laparoscopic and open tension-free repair of recurrent inguinal hernias: a prospective randomized study. Surgical Endoscopy And Other Interventional Techniques. 2006; 20 (7):1099-1104.

[20] Günal Ö., Özer Ş., Gürleyik E., Bahçebaşı T. Does the approach to the groin make a difference in hernia repair? Hernia: the journal of hernias and abdominal wall surgery. 2007; 11 (5):429-434.

[21] More Dr. Milind P., Nasta Dr. Amrit Manik, More Dr. Rakhi M., Shedg Dr. Rakesh. Comparison of Laparoscopic TAPP (Transabdominal Preperitoneal) and Laparoscopic TEP (Totally Extra peritoneal) Techniques for Inguinal Hernia Repair- An Observational Study of 60 Cases. IOSR Journal of Dental and Medical Sciences (IOSR-JDMS). 2016; 15 (7):9093.

[22] Bansal V. K., Misra M. C., Babu D., Victor J., Kumar S., Sagar R., Rajeshwari S., Krishna A., Rewari V. A prospective, randomized comparison of long-term outcomes: chronic groin pain and quality of life following totally extraperitoneal (TEP) and transabdominal preperitoneal (TAPP) laparoscopic inguinal hernia repair. Surgical endoscopy. 2013; 27 (7):23732382 .

[23] Verma Nimesh, Prajapati Keyur, Mistry Siddharth, Bagmar Gaurav, Kumar Ajay, Patel Dharmik. A comparative study between total extraperitoneal (TEP) repair and Transabdominal pre-peritoneal (TAPP) repair in management of inguinal hernia. National Journal Of Medical Research. 2015;5:64-66.

[24] Gong K., Zhang N., Lu Y., Zhu B., Zhang Z., Du D., Zhao X., Jiang H. Comparison of the open tension-free mesh-plug, transabdominal preperitoneal (TAPP), and totally extraperitoneal (TEP) laparoscopic techniques for primary unilateral inguinal hernia repair: a prospective randomized controlled trial. Surgical endoscopy. 2011; 25 (1):234-239.

[25] Pokorny H., Klingler A., Schmid T., Fortelny R., Hollinsky C., Kawji R., Steiner E., Pernthaler H., Függer R., Scheyer M. Recurrence and complications after laparoscopic versus open inguinal hernia repair: results of a prospective randomized multicenter trial. Hernia: the journal of hernias and abdominal wall surgery. 2008;12.
[26] He Zirui, Hao Xiaohui, Li Jianwen, Zhang Yun, Feng Bo, Yue Fei, Xue Pei, Gong Hangjun. Laparoscopic inguinal hernia repair in elderly patients: single center experience in 12 years. Annals of Laparoscopic and Endoscopic Surgery. 2017; 2 (1).

[27] Neumayer L., Giobbie-Hurder A., Jonasson O., Fitzgibbons R., Jr., Dunlop D., Gibbs J., Reda D., Henderson W. Open mesh versus laparoscopic mesh repair of inguinal hernia. $\mathrm{N}$ Engl J Med. 2004; 350 (18):1819-1827.

[28] Lau H., Patil N. G., Yuen W. K., Lee F. Prevalence and severity of chronic groin pain after endoscopic totally extraperitoneal inguinal hernioplasty. Surgical endoscopy. 2003; 17 (10):1620-1623.

[29] Haidenberg Jaime, Kendrick Michael L., Meile Tobias, Farley David R. Totally extraperitoneal (TEP) approach for inguinal hernia: the favorable learning curve for trainees. Current Surgery. 2003; 60 (1):65-68.

[30] Wake Beverly L, McCormack Kirsty, Fraser Cynthia, Vale Luke, Perez Juan, Grant Adrian. Transabdominal pre peritoneal (TAPP) vs totally extraperitoneal (TEP) laparoscopic techniques for inguinal hernia repair. The Cochrane Library. 2005.

[31] Schmedt C-G, Däubler P, Leibl BJ, Kraft K, Bittner R. Simultaneous bilateral laparoscopic inguinal hernia repair. Surgical endoscopy. 2002; 16 (2):240-244.

[32] Kapiris SA, Brough WA, Royston CMS, O'Boyle C, Sedman PC. Laparoscopic transabdominal preperitoneal (TAPP) hernia repair. Surgical endoscopy. 2001; 15 (9):972-975.

[33] Leibl Bernhard J, Schmedt Claus-Georg, Schwarz Jochen, Däubler Peter, Kraft Klaus, Schloßnickel Barbara, Bittner Reinhard. A single institution's experience with transperitoneal laparoscopic hernia repair. The American journal of surgery. 1998; 175 (6):446-452.

[34] Bittner R, Schmedt C-G, Schwarz J, Kraft K, Leibl BJ. Laparoscopic transperitoneal procedure for routine repair of groin hernia. British journal of surgery. 2002; 89 (8):10621066 .

[35] Misra Mahesh C., Kumar Sareesh, Bansal Virinder K. Total extraperitoneal (TEP) mesh repair of inguinal hernia in the developing world: comparison of low-cost indigenous balloon dissection versus direct telescopic dissection: a prospective randomized controlled study. Surgical endoscopy. 2008; 22 (9):1947. 